Positive mental wellbeing in Australian adolescents:

Evaluating the Warwick-Edinburgh Mental Wellbeing Scale

\author{
Simon C. Hunter \\ School of Psychological Sciences and Health, \\ University of Strathclyde \\ Stephen Houghton \\ Graduate School of Education, \\ The University of Western Australia \\ $\&$ \\ Lisa Wood \\ Centre for the Built Environment and Health, \\ The University of Western Australia
}

Key words: Wellbeing; WEMWBS; Adolescence

Running head: Positive Mental Wellbeing in Adolescents

Correspondence: Dr Simon C. Hunter,

School of Psychological Sciences and Health,

40 George Street,

University of Strathclyde,

Glasgow, UK.

Email: simon.hunter@strath.ac.uk.

Tel. (+44) 1415484879

Fax: (+44) 1415484001

Please cite as:

Hunter, S.C., Houghton, S., Wood, L. (in press). Positive mental wellbeing in Australian adolescents: Evaluating the Warwick-Edinburgh Mental Wellbeing Scale. Australian Educational and Developmental Psychologist. 
Positive mental wellbeing in Australian adolescents:

Evaluating the Warwick-Edinburgh Mental Wellbeing Scale

\begin{abstract}
While there is increasing recognition of the need to go beyond measures of mental ill health, there is a relative dearth of validated tools for assessing mental wellbeing among adolescents. The Warwick-Edinburgh Mental Wellbeing Scale (WEMWBS) is a promising tool for use in this context, and this study evaluated its use in an Australian context. The WEBWBS was completed by 829 Western Australian adolescents, aged 13 to 16 years old, drawn from grades 8, 9 and 10 in seven separate high schools. Using confirmatory factor analytic techniques, the utility of the full 14item scale was not supported but good fit for a previously validated seven-item short version (SWEMWBS) was supported. Strong measurement invariance was demonstrated across age and weak measurement invariance was demonstrated across gender. The scale has good internal reliability. There were no differences in SWEMWBS scores across Grades 8 to 10. Overall, the SWEMWBS represents a useful tool for educational, developmental, and school psychologists investigating positive mental wellbeing in younger adolescents.
\end{abstract}


Adolescence is a critical developmental period for mental health, with symptoms of lifelong mental illness typically developing prior to age 25 years (Call, Riedel, Hein, McLoyd, Peterson, \& Kipke, 2002). Current reported rates of mental health problems among adolescents are high, with prevalence estimates reported to be $40.3 \%$ over 12 months and $23.4 \%$ over the past 30 days (Kesslet, Avenevoli, Costello, Georgiades, Green, Gruber et al., 2012). Almost 20\% of young people reporting mental health problems report three or more problems (Heneghan, Stein, Hurlburt, Zhang, Rolls-Reutz, Fisher, Landsverk, \& Horowitz, 2013). Such problems are more prevalent in rural areas as a result of psychosocial, behavioural and demographic variables which differ from those in urban areas (see Australian Bureau of Statistics, 2012). The Australian landscape makes such issues especially salient for professionals working in the country. The imposing burden on society in terms of the individual, families and the health, justice and welfare systems as a consequence of mental health problems that arise in childhood and adolescence are well documented (Heckman, 2012), making adolescence a critical time for developing positive mental wellbeing.

Undeniably, positive mental wellbeing has emerged as an important protective factor against mental health issues (Gargiulo \& Stokes, 2009) and a range of other health outcomes (Australian Bureau of Statistics, 2008). Consequently, because of its major implications for health and social outcomes and psychological functioning (Linley \& Joseph, 2004; Stewart-Brown, Tennant, Tennant, Platt, Parkinson, \& Weich, 2009), and for its contribution to public health and policy issues (Dolan, Laylard, \& Metcalf, 2011), positive mental wellbeing has received attention worldwide. In many countries, promoting positive mental wellbeing among teenagers has become a national priority (Clarke, Friede, Putz, Ashdown, Martin, Blake et al., 2011), particularly as a preventive and population based complement to the treatment of psychopathology (Jacka, Reavley, Jorm, Toumboiurou, Lewis, Berk et al., 2013; Perry, Presley-Cantrell, \& Dhingra, 2010).

Considered by many to be a complex construct, positive mental wellbeing covers both hedonic (i.e., happiness, subjective wellbeing) and eudemonic (i.e., positive functioning) aspects of 
wellbeing (Clarke et al., 2011; Ryan \& Deci, 2001; Tennant, Hiller, Fishwick, Platt, Joseph, Weich et al., 2007), both of which together comprise the broad concept of mental wellbeing (Clarke et al., 2011). It has also been argued that it takes a combination of these for one to be considered mentally healthy (Keyes, 2007). However, regardless of the increased interest in positive mental wellbeing, there is a dearth of appropriate measures (Hu, Stewart-Brown, Twigg, \& Weich, 2007), particularly for adolescents. Consequently, surveys and intervention evaluations have relied on measures of mental illness (e.g., prevalence of depression or anxiety) as their 'marker' of mental wellbeing (Hu et al., 2007), even though mental wellbeing is conceptualised as more than the absence of mental illness (Clarke et al., 2011).

One instrument that has shown promise and may be particularly useful for school, educational, and developmental psychologists is the 14-item Warwick-Edinburgh Mental Well Being Scale (WEMWBS: Stewart-Brown et al., 2011; Tennant et al., 2007). This covers both hedonic elements of positive mental wellbeing ( 9 items covering happiness, joy, contentment: e.g., "I’ve been feeling cheerful", "I've been feeling optimistic about the future", "I've been feeling relaxed") and eudemonic elements of positive mental wellbeing ( 5 items covering psychological functioning, autonomy, positive relationships with others, sense of purpose in life: e.g., "I've had energy to spare", "I've been thinking clearly", "I've been able to make up my own mind about things"). For a description of its development see Tennant et al. (2007a; 2007b). However, the data supporting its use come almost exclusively from adults: Cronbach alphas of $\alpha=0.87-0.93$ and significant correlations with other scales measuring components of affect or wellbeing (Clarke et al., 2011; Lloyd \& Devine, 2012; Tennant et al., 2007a) including using the shorted 7 item version (Vaingankar, Subramaniam, Chong, Abdin, Edelen, Picco et al., 2011); significant age and gender effects (Tennant et al., 2007a); and test-retest reliability 0.66 - 0.83 (Clarke et al., 2011; Tennant et al., 2007a). Two studies employing the Rasch model (Rasch, 1960, 1980) have also found support for the unidimensionality of the WEMWBS, but in the form of a shortened 7-item version (Bartram, Sinclair, \& Baldwin, 2012; Stewart-Brown et al., 2009). Finally, Maheswaran, Weich, Powell, and 
Positive Mental Wellbeing in Adolescents Stewart-Brown (2012) report that the WEMWBS is sensitive to a range of interventions across a number of different populations. Thus, items in the WEMWBS appear suitable for measuring the latent trait of positive mental wellbeing in adults.

To date, however, the administration of the WEMWBS with adolescents has been scarce, with it seems only one study conducted. Employing Confirmatory Factor Analysis (CFA) with data from 1650, 13 to 16 year olds, Clarke et al. (2011) reported excellent fit statistics (GFI = 1.000, RMSEA $=0.003)$. Although there was strong support for a single underlying construct for the 14 -item WEMWBS, follow up single sex focus group interviews with 80 of the adolescent participants identified issues pertaining to: definition and understanding of items, potential for misinterpretation of items, items possibly causing embarrassment, and variable interpretation where items referred to a more holistic reflective approach to oneself. Although some of the participants thought there "might be some redundancy which could be removed through amalgamation of items" (Clarke et al., 2011, p. 7), the authors provided no indication of which items the participants were referring to.

The promotion of mental wellbeing in adolescents is a high priority globally, but is hindered by the dominance of measures of mental illness and poor psychological wellbeing. While the WEMWBS with its orientation towards protective factors for mental wellbeing (Stewart-Browne 2011) is a promising exception, there is a need to trial it in different populations (Vainganker et al., 2011) and to test its validity in other countries where concepts of mental wellbeing may be unique and culturally specific (Tennant et al., 2007a).

\section{Aims and objectives}

Our main objective was to test the fit of the factor structure (the full 14-item scale and the shortened 7-item scale) of the WEMWBS with a sample of Australian adolescents. Specific aims were to assess (i) the extent to which the measure is equivalent across males and females and across three adolescent school stages and (ii) the effects of these two variables (gender, school stage) on the measure's scores. As the result of a rigorous evaluation of the WEMWBS, school, educational 
and developmental psychologists may have access to an instrument that measures positive mental wellbeing in young people.

\section{Participants and settings}

\section{Method}

The sample comprised 829 adolescents aged 13 to 16 years randomly selected from seven separate high schools. Of the 829, 391 (196 males, 195 females) were in Grade 8 (age 13 years), 290 (131 males, 159 females) in Grade 9 (age 14 years), and 138 (66 males, 72 females) in Grade 10 (age 15-16 years). All participating high schools $(n=7)$ were located across different socioeconomic status (SES) areas as indexed by their postal codes from the Socio-Economic Indexes for Areas within Western Australia (Australian Bureau of Statistics, 2008). Two were in high SES, 2 in middle SES and 1 in a low SES), located in the metropolitan area of the Western Australian capital city of Perth. Of the remaining two schools, one was a District High School located on the boundaries of the metropolitan and rural regions in a middle SES area and the other was located in a rural area.

\section{Instrumentation}

The Warwick-Edinburgh Mental Well Being Scale (WEMWBS: Tennant et al., 2007a, b) was specifically developed to assess positive mental wellbeing at a general population level. It comprises 14 positively worded items to which participants respond using a five point Likert scale (scored 1 "none of the time", 2 "rarely", 3 "some of the time", 4 "often", 5 "all of the time"), thereby providing a total score of 14 to 70 . Responses are based on a participant's feelings over the previous two weeks. Higher levels of positive mental wellbeing are indicated by higher scores.

\section{Procedure}

Permission to conduct the research was initially obtained from the Human Research Ethics Committee of the administering institution and the Western Australian Department of Education. It adhered to all legal requirements of the study country. Following this, 12 schools were randomly selected from a mix of socioeconomic and metropolitan/rural areas. Specifically, three schools in each of high SES, middle SES and low SES locations were randomly identified from the Western 
Australian Department of Education schools online website. Two rurally located schools were also randomly selected along with one District high school. The principal of each of these schools was then contacted by telephone to ascertain their interest in participating in the research. Information sheets explaining the research, along with consent forms for parents, were delivered to the eight schools agreeing to participate in the study. One rural school subsequently decided not to participate leaving seven schools. The participating metropolitan based schools were all within $30 \mathrm{kms}$ of the city, while the District High school was approximately $50 \mathrm{kms}$ away, and the rural school was some $2,000 \mathrm{kms}$ out of the city. The sample of 829 students represented an affirmative return rate of $69 \%$.

The WEMWBS was administered to the participants in groups of approximately 20-25 students during their regular class time by school personnel who had been nominated by the principals to liaise with the researchers. Each scale administrator was provided with a written set of instructions to ensure standardization of administration. Prior to completing the instrument participants were informed of the nature of the research and were assured of the anonymity of their responses.

\section{Results}

Confirmatory factor analyses were conducted to examine the fit of the full single-factor 14-item scale and the shortened single-factor 7-item scale. AMOS 21.0 was used to perform these analyses, and we used four indices to assess the goodness of fit of a first-order measurement model: the comparative fit index (CFI and TLI: above .95 indicates good fit, above .90 indicates adequate fit), the root mean-square error or approximation (RMSEA: .05 or less indicates good fit, .08 or less indicates adequate fit), standardized root mean square residual (SRMR: .08 or below indicates good fit), the CMIN/DF (lower than 2-3 indicates good fit) (Carmines \& McIver, 1981; Keith, 2006) and chi-square (non-significant values represent good fit). Once the best fitting measurement model was identified, the measurement invariance of this was compared across gender and age using the change in CFI and by investigating the RMSEA statistics and associated $90 \%$ confidence intervals. Finally, age differences in participants' scores were evaluated.

Evaluation of Full 14-Item and Shortened 7-Item Scales. 
The full 14-item measurement model demonstrated marginal fit: $\chi^{2}(\mathrm{df}=77)=492.33, p<.001$, $\mathrm{CMIN} / \mathrm{DF}$ ratio $=6.39, \mathrm{CFI}=.93, \mathrm{TLI}=.92, \mathrm{RMSEA}=.080(90 \%$ confidence interval $[\mathrm{CI}]: .074$, $.087)$, SRMR $=.041$. See Figure 1 for standardized estimates. Examination of modification indices suggested that correlating the errors for items 7 ("I've been thinking clearly") and 12 ("I've been feeling loved") would make the largest difference to model fit. However, we could see no conceptual justification for correlating these items, and doing so made little difference to the fit anyway, $\chi^{2}(\mathrm{df}=76)=475.25, p<.001, \mathrm{CMIN} / \mathrm{DF}$ ratio $=6.25, \mathrm{CFI}=.94, \mathrm{TLI}=.92, \mathrm{RMSEA}=$ .079 (90\% CI: .072, .086), SRMR $=.040$.

Figure 1 about here

The 7-item model demonstrated better fit: $\chi^{2}(\mathrm{df}=14)=64.99, p<.001, \mathrm{CMIN} / \mathrm{DF}$ ratio $=4.64$, $\mathrm{CFI}=.98, \mathrm{TLI}=.97, \mathrm{RMSEA}=.066(90 \% \mathrm{CI}: .050, .083), \mathrm{SRMR}=.027$. Modification indices suggested that the errors for items 6 ('I've been dealing with problems well") and 7 ("I've been thinking clearly") should be correlated, and based on face content there appeared some conceptual justification for making such a modification. This change resulted in a good fitting model: $\chi^{2}(\mathrm{df}=$ $13)=30.75, p=.004, \mathrm{CMIN} / \mathrm{DF}$ ratio $=2.37, \mathrm{CFI}=.99, \mathrm{TLI}=.99, \mathrm{RMSEA}=.040(90 \% \mathrm{CI}: .022$, $.059)$, SRMR $=.020$, and was accepted as the final model. Internal reliability (Cronbach's Alpha) of the scale was .87 , indicating good internal reliability. See Figure 2 for standardized estimates.

Figure 2 about here

\section{Invariance of the 7-Item Scale Across Gender and School Stage.}

To assess factorial invariance, we compared three models. The first model was the unconstrained model in which the same factor structure was present for the competing groups, but no further constraints were placed upon the model. The second model was the weak factorial model, where the addition of constraints upon the factor loadings was added. Finally, the third model was the strong factorial model and included the additional constraint that indicator intercepts also be equal. Strong factorial invariance indicates that slopes and intercepts are equal across groups and that the factor scores are comparable across groups (Little, Card, Slegers, \& Ledford, 2007). Following Little et al. 
(2007), we did not use change in chi-square as an indicator of invariance because of its documented sensitivity to sample size. Rather, we used change in CFI $(\triangle \mathrm{CFI})$ as one indicator of invariance $(\triangle \mathrm{CFI}>-.01$ indicates violation of invariance) and whether the invariance model's $90 \%$ RMSEA confidence intervals included the RMSEA of the unconstrained model.

For gender, these analyses indicated support for weak factorial invariance but not strong factorial invariance (see Table 1). In contrast, there was support for strong invariance with respect to school stage.

Table 1 about here

These results indicate that the 7-item WEMWBS may be used to compare developmental trends in positive mental wellbeing from Grades 8 to 10 , but that its psychometric properties do not support use of the measure for investigating gender differences.

\section{Effect of School Stage on 7-Item WEMWBS Score.}

Using the formula $\mathrm{W}=\mathrm{BS}^{-1}$, where $\mathrm{B}$ is the matrix of covariances between the unobserved and observed variables, and $\mathrm{S}$ is the matrix of covariances among the observed variables, AMOS 21.0 calculated factor score weights for each of the items based on the accepted measurement model. To use these, each participant's score on each item was multiplied by the factor score weight for that item, and this was then added to a similar score for the following item, and so on. Factor score weights are shown in Table 2, and factor score means and standard deviations are shown by school stage in Table 3.

Table 2 about here

To examine the effects of school stage, a one-way independent ANOVA was conducted. This was not significant, $F(2,823)=1.08, p=.341$, indicating that there are no differences in SWEMWBS scores across Grades 8 to 10.

Table 3 about here

\section{Discussion}


Positive Mental Wellbeing in Adolescents

Valid measures of positive mental wellbeing that can be used with adolescent populations are needed. They need to be tested in different cultural contexts to enhance their generalisability. The primary aim of this research was to test the fit of the factor structure (the full 14-item scale and the shortened 7-item scale) of the WEMWBS with Australian adolescents. There has been only one published study and this was undertaken with Scottish teenagers.

More specifically, the study aimed to assess (i) the extent to which the measure is equivalent across males and females and across three adolescent school stages and (ii) the effects of these two variables (gender, school stage) on the measure's scores. To date, limited psychometric data exist on measures of positive mental wellbeing in mainstream community adolescents. This is an important issue to be addressed given that adolescence is an important formative developmental stage that lays the groundwork for well-being and mental health in adulthood (Ford, Goodman, \& Meltzer, 2003). Mental wellbeing has the potential to improve quality of life, prevent mental and physical illness, and reduce the use of health services (Keyes, 2007; Keyes, Dhingra, \& Simoes, 2010). There is clearly a need for validated measures that can be used in adolescent level studies.

Given the dearth of adolescent population studies employing measures for positive mental wellbeing, comparisons with the present data are difficult to undertake. Unlike the study conducted with 13-16 year old adolescents by Clarke et al. (2011) (fit statistics: GFI = 1.000, RMSEA = 0.0032) which supported a single underlying construct for the 14-item WEMWBS, the model fit in the present study, while acceptable, was not so supportive $(\mathrm{CFI}=.93$, RMSEA $=.080)$.

Even though Clarke et al. (2011) obtained excellent model fit statistics, issues of item redundancy still arose. The issue of less than perfect model fit has arisen in a number of studies employing CFA and Rasch model analysis, but these studies were with adult populations in different countries (Gremigni, \& Stewart-Brown, 2011; Lloyd \& Devine, 2012; López et al., 2013; Tennant et al., 2007a). Taken together, these studies demonstrate the importance of examining the psychometric properties of an instrument in different populations and in different settings (see Vaingankar et al., 2011). 
Positive Mental Wellbeing in Adolescents

While it has been proposed that mental wellbeing is unique and culturally specific (Tennant et al., 2007a) it is not yet clear why results from the present study with Australian adolescents returned different results to Clarke et al.'s. (2011) Scottish and English sample. One difference between the current study and Clarke et al.'s is that our study intentionally sampled from rural areas whereas Clark et al. sampled from two cities. This may be pertinent because (in Australia at least) the experiences of rural and urban groups appear to differ in terms of educational, recreational and employment opportunities. Lack of opportunity may contribute toward greater risk-taking behaviour among young people from rural areas (Quine et al., 2003), possibly leading to greater focus upon hedonic rather than eudemonic aspects of wellbeing. Such differences in life-chances may therefore impact upon the ways in which happiness and wellbeing and conceptualised in these groups. Future research should seek to investigate this issue further.

Tennant et al. (2007a, p. 10) suggested that although the 14-item WEMWBS was short, acceptable and meaningful to general population groups, it "could be shortened further." Thus, in the present study we also tested the fit of the seven item short version of the WEMWBS (i.e., the SWEMWBS, see Bartram et al., 2012; Stewart-Brown et al., 2009) and this demonstrated superior model fit to the 14-item version. Although Bartram et al. and Stewart-Brown et al. also found improved fit using the shortened version, the latter raised the point that most of the items retained in the shortened version represented aspects of psychological and eudemonic wellbeing, with fewer covering hedonic wellbeing or affect. This is in contrast to the broad consensus that mental wellbeing is a complex construct covering both hedonic and eudemonic wellbeing (Clarke et al., 2011; Ryan \& Deci, 2001; Tennant et al., 2007a). The SWEMWBS may therefore be assessing a narrower conceptualisation of positive mental wellbeing than the full WEMWBS, one which reflects a focus upon general wellbeing rather than a subjective happiness based on accumulated moments of pleasure (see Ryan \& Deci, 2001).

The present study one-factor model represented by seven items was invariant across school stage. This indicates that the meaning and interpretation of scale scores is equivalent across Grades 
8 to 10 . We also found that actual scale scores were not different for young people across this grade range. We were unable to examine gender differences on scale scores because the measure was not sufficiently psychometrically robust to permit such an analysis. There was a clear drop in fit when considering strong factorial invariance, indicating that factor scores for boys and girls cannot be considered to be comparable (Wu \& Zumbo, 2007). This is a weakness of the SWEMWBS, and may call into question results from previous studies which have reported gender differences in positive mental wellbeing (Clarke et al., 2011; Frick, Fitzgerald, Shiely, \& Perry, 2013; Tennant et al., 2007a). Future research should seek to identify new items, or modify the existing items, so that the measure allows better investigation of potential gender differences. The results from Clarke et al.'s (2011) focus groups may help guide such work as these identified items which girls and boys felt more confident answering, as well as items which young people felt could be ambiguous (e.g., "I've been feeling close to other people" could be understood as referring to romantic or sexual relationships).

In Australia (Dobia \& O’Rourke, 2011; Scott, 2011) and beyond (Clarke et al., 2011) the importance of attending to young people's positive mental health as well as reducing levels of negative wellbeing is increasingly emphasised. Despite the limitations noted above, the SWEMWBS is likely to prove a useful tool for educational, developmental and school psychologists, and for other professionals working in this area. The measure reported here allows professionals to assess levels of wellbeing using a reliable measure and to compare levels of wellbeing across, at least, Grades 8 to 10 . Care should be taken when comparing boys' and girls' scores because differences between them may be misleading.

It must be acknowledged that our results are based solely on self-report data. Corroborative information such as file data and observations might enhance reliability. However, there is clear evidence that self-report is an effective means of obtaining an accurate insight into the subjective dispositions (such as positive mental wellbeing) that can be difficult to obtain from third parties such as teachers and parents (see Frick, Barry, \& Kamphaus, 2009). Moreover, the validity of self- 
Positive Mental Wellbeing in Adolescents report on psychopathology and personality tends to increase from childhood to adolescence whereas the validity of parental and teacher reports decreases for this period (Essau, Sasagawa, \& Frick, 2006). There was no demonstration of convergent and discriminant validity in the present study. This must be a focus of future research if the 7- and 14-item versions of the WEMWBS are to be used as indicators of the mental wellbeing of adolescents, for monitoring population level changes in mental wellbeing, and as an outcome measure in interventions.

In conclusion, the 14-item WEMWBS shows marginal model fit with adolescents. The shortened version (i.e., 7-item SWEMWBS) demonstrates improved fit, along with high levels of internal reliability and strong factor invariance across Grades 8 to 10. The weakness of the short form was that its factor structure was not invariant across gender. Positive mental wellbeing has the potential to improve quality of life and prevent mental and physical illness (Keyes, 2007; Keyes, Dhingra, \& Simoes, 2010). Given the need for validated measures of positive mental wellbeing that can be used in adolescent population level studies, the present findings indicate that the SWEMWBS is a concise and suitable measure. 
Positive Mental Wellbeing in Adolescents

\section{References}

Australian Bureau of Statistics. (2008). An introduction to socio-economic indexes for areas (SEIFA), 2006. Canberra: Author.

Australian Bureau of Statistics. (2012). ABS 3303.0. Causes of Death, Australia, 2010. ABS: Canberra.

Bartram, D. J., Sinclair, J. M. \& Baldwin, D. S. (2012). Further validation of the WarwickEdinburgh Mental Well-being Scale (WEMWBS) in the UK veterinary profession: Rasch analysis. Quality of Life Research, 22(2), 379-391.

Call, K., Riedel, A. A., Hein, K., McLoyd, V., Petersen, A., \& Kipke, M. (2002). Adolescent health and well-being in the twenty first century: A global perspective. Journal of Research on Adolescence, 12(1), 69-98.

Carmines, E. G., \& McIver, J. P. (1981). Analyzing models with unobserved variables: Analysis of covariance structures. In G. W. Bohrstedt \& E. F. Borgatta (Eds.), Social measurement: Current issues (pp. 65-115). Beverly Hills, CA: Sage.

Clarke, A., Friede, T., Putz, R., Ashdown, J., Martin, S., Blake, A., ..... Platt, S. (2011). WarwickEdinburgh Mental Well-being Scale (WEMWBS): Validated for teenage school students in England and Scotland. A mixed methods assessment. BMC Public Health, 11(1), 487.

Davoren, M. P., Fitzgerald, E., Shiely, F., \& Perry, I. J. (2013). Positive mental health and wellbeing among a third level student population. PLOS ONE 8(8): e74921.

Dobia, B. \& O'Rourke, V. G. (2011). Promoting the mental health and wellbeing of Indigenous children in Australian primary schools. Canberra: Commonwealth of Australia.

Dolan, P., Layard, R., \& Metcalf, R. (2011). Measuring subjective well-being for public policy. UK: Office for National Statistics Report.

Essau, C. A., Sasagawa, S., \& Frick, P. J. (2006). Callous-unemotional traits in a community sample of adolescents. Assessment, 13(4), 454-469. 
Positive Mental Wellbeing in Adolescents

Ford, T., Goodman, R., \& Meltzer, H. (2003). The British child and adolescent mental health survey 1999: the prevalence of DSM-IV disorders. Journal of the American Academy of Child and Adolescent Psychiatry, 42, 1203-1211.

Frick, P. J., Barry, C. T., \& Kamphaus, R.W. (2009). Clinical assessment of child and adolescent personality and behaviour (3rd ed). New York: Springer.

Gargiulo, R. A. \& Stokes, M. A. (2009). Subjective well-being as an indicator for clinical depression. Social Indicators Research, 92, 517-527.

Gremigni, P. \& Stewart-Brown, S. L. (2011). Measuring mental well-being: Italian validation of the Warwick-Edinburgh Mental Well-Being Scale (WEMWBS). Giornale Italiano di Psicologia, 2, 485-508.

Heneghan, A., Stein, R., Hurlburt, M. S., Zhang, J., Rolls-Reutz, J., Fisher, E., Landsverk, J., \& Horwitz, S. (2013). Mental health problems in teens investigated by U.S. child welfare agencies. Journal of Adolescent Health, 52(5), 634 - 640.

Heckman, J. J. (2012). The developmental origins of health. Health Economics, 21, 24 - 29.

Hu, Y., Stewart-Brown, S., Twigg, L., \& Weich, S. (2007). Can the 12 item General Health Questionnaire be used to measure positive mental health? Psychological Medicine, 37(7), 10051013.

Jacka, F. N., Reavley, N. J., Jorm, A. F., Toumboiurou, J., Lewis, A. J., \& Berk, M. et al. (2013). Prevention of common mental disorders: What can we learn from those who have gone before and where do we go next? Australian and New Zealand Journal of Psychiatry, 47, 920-929. 
Positive Mental Wellbeing in Adolescents

Keith, T. Z. (2006). Multiple regression and beyond. USA: Routledge.

Kessler, R., Avenevoli, S., Costello, E. J., Georgiades, K., Green, J., Gruber, M. J., et al. (2012). Prevalence, persistence, and sociodemographic correlates of $D S M-I V$ disorders in the National Comorbidity Survey Replication Adolescent Supplement. Archives General Psychiatry, 69(4), 372-380.

Keyes, C. L. (2007). Promoting and protecting mental health as flourishing: a complimentary strategy for improving national mental health. American Psychologist, 62, 95-108.

Keyes, C. L., Dhingra, S. S., \& Simoes, E. J. (2010). Change in levels of positive mental health as a predictor of future risk of mental illness. American Journal of Public Health, 100, 2366-2371.

Linley, P. A. \& Joseph, S. (2004). Positive psychology in practice. Hoboken, NJ: Wiley.

Little, T. D., Card, N. A., Slegers, D. W., \& Ledford, E. C. (2007). Representing contextual effects in multiple-group MACS models. In T. Little, J. A Bovaird, \& N. A. Card (Eds.), Modeling contextual effects in longitudinal studies (pp. 121-147). New Jersey: LEA.

Lloyd, K., \& Devine, P. (2012). Psychometric properties of the Warwick-Edinburgh Mental Wellbeing Scale (WEMWBS) in Northern Ireland. Journal of Mental Health, 21(3), 257-263.

López, M., Gabilondo, A., Codony, M., García-Forero, C., Vilagut, G., Castellví, P., ...... Alonso, J. (2013). Adaptation into Spanish of the Warwick-Edinburgh Mental Well-being Scale (WEMWBS) and preliminary validation in a student sample. Quality of Life Research, 22, 1099-1104.

Maheswaran, H., Weich, S., Powell, J., \& Stewart-Brown, S. (2012). Evaluating the responsiveness of the Warwick Edinburgh Mental Well-Being Scale (WEMWBS): Group and individual level analysis. Health and Quality of Life Outcomes, 10(1), 156.

Perry, G., Presley-Cantrell, L., \& Dhingra, S. (2010). Addressing mental health promotion in chronic disease prevention and health promotion. American Journal of Public Health, 100(12), 2337-2339. 
Quine, S., Bernard, D., Booth, M., Kang, M., Usherwood, T., Alperstein, G., \& Bennett, D. (2003). Health and access issues among Australian adolescents: a rural-urban comparison. Rural and Remote Health, 3(3), 245.

Rasch, G. (1960). Probabilistic models for some intelligence and attainment tests. Copenhagen: Danish Institute for Educational Research.

Rasch, G. (1980). Probabilistic models for some intelligence and attainment tests (Expanded). Chicago: University of Chicago Press.

Ryan, R. M. \& Deci, E. L. (2001). On happiness and human potentials: A review of research on hedonic and eudaimonic well-being. Annual Review of Psychology, 52(1), 141-166.

Scott, M. (2011). Report of the Inquiry into the mental health and wellbeing of children and young people in Western Australia. Perth, Western Australia: Commissioner for Children and Young People. References, 296.

Stewart-Brown, S. L., Platt, S., Tennant, A., Maheswaran, H., Parkinson, J., Weich, S., ... \& Clarke, A. (2011). The Warwick-Edinburgh Mental Well-being Scale (WEMWBS): a valid and reliable tool for measuring mental well-being in diverse populations and projects. Journal of Epidemiology and Community Health, 65(Suppl 2), A38-A39.

Stewart-Brown, S., Tennant, A., Tennant, R., Platt, S., Parkinson, J., \& Weich, S. (2009). Internal construct validity of the Warwick-Edinburgh Mental Well-being Scale (WEMWBS): A Rasch analysis using data from the Scottish Health Education Population Survey. Health and Quality of Life Outcomes, 7, 15-22.

Tennant, R., Hiller, L., Fishwick, R., Platt, S., Joseph, S., Weich, S., ...... Stewart-Brown, S. (2007a). The Warwick-Edinburgh Mental Well-being Scale (WEMWBS): development and UK validation. Health and Quality of Life Outcomes, 5, 63.

Tennant, R., Joseph, S., \& Stewart-Brown, S. (2007b). The Affectometer 2: A measure of positive mental health in UK populations. Quality Life Research, 16(4), 687-695. 
Positive Mental Wellbeing in Adolescents

Vaingankar, J. A., Subramaniam, M., Chong, S. A., Abdin, E., Edelen, M. O., Picco, L., ......

Sherbourne, C. (2011). The positive mental health instrument: development and validation of a culturally relevant scale in a multi-ethnic Asian population. Health and Quality of Life Outcomes, 9(92), 1-18.

Wu, A. D., Li, Z., \& Zumbo, B. D. (2007). Decoding the meaning of factorial invariance and updating the practice of multi-group confirmatory factor analysis: A demonstration with TIMSS data. Practical Assessment, Research \& Evaluation, 12(3), 1-26. 
Acknowledgements: The authors would like to extend their thanks to all the young people who so willingly took part in this study. We would also like to thank the schools and staff in those schools for their help and support throughout data collection.

Financial support: This research was funded in part by the Western Australian Health Promotion Foundation (Healthway) and The Australian Research Council. The Warwick-Edinburgh Mental Well-being Scale was funded by the Scottish Executive National Programme, commissioned by NHS Health Scotland, developed by the University of Warwick and the University of Edinburgh, and is jointly owned by NHS Health Scotland, the University of Warwick and the University of Edinburgh.

Conflicts of interest: None of the authors have any conflicts of interest to declare.

Ethical standards: The authors assert that all procedures contributing to this work comply with the ethical standards of the relevant national and institutional committees on human experimentation and with the Helsinki Declaration of 1975, as revised in 2008. 
Positive Mental Wellbeing in Adolescents

Table 1.

Fit Indices Used for Assessing Factor Invariance Across Gender and School Stage.

\begin{tabular}{ccccc}
\hline \multicolumn{1}{c}{ Model } & \multicolumn{2}{c}{ CFI } & \multicolumn{2}{c}{ RMSEA (90\% CI) } \\
& Gender & School Stage & Gender & School Stage \\
\hline Model 1 (Unconstrained) & .988 & .984 & $.036(.022, .049)$ & $.027(.017, .037)$ \\
Model 2 (Weak invariance) & .989 & .980 & $.031(.017, .044)$ & $.029(.019, .038)$ \\
Model 3 (Strong invariance) & .959 & .977 & $.053(.043, .063)$ & $.029(.020, .038)$ \\
\hline
\end{tabular}


Table 2.

Factor Score Weights

Item Factor Weight

1. I have been feeling optimistic about the future .155

2. I've been feeling useful .144

3. I've been feeling relaxed .104

6. I've been dealing with problems well .072

7. I've been thinking clearly .119

9. I've been feeling close to other people .100

11. I've been able to make up my own mind about things .111 
Table 3.

Means (and Standard Deviations) by School Stage

School Stage Mean (Standard Deviation)

Grade $8 \quad 2.86(0.61)$

Grade $9 \quad 2.82(0.62)$

Grade $10 \quad 2.78(0.64)$ 


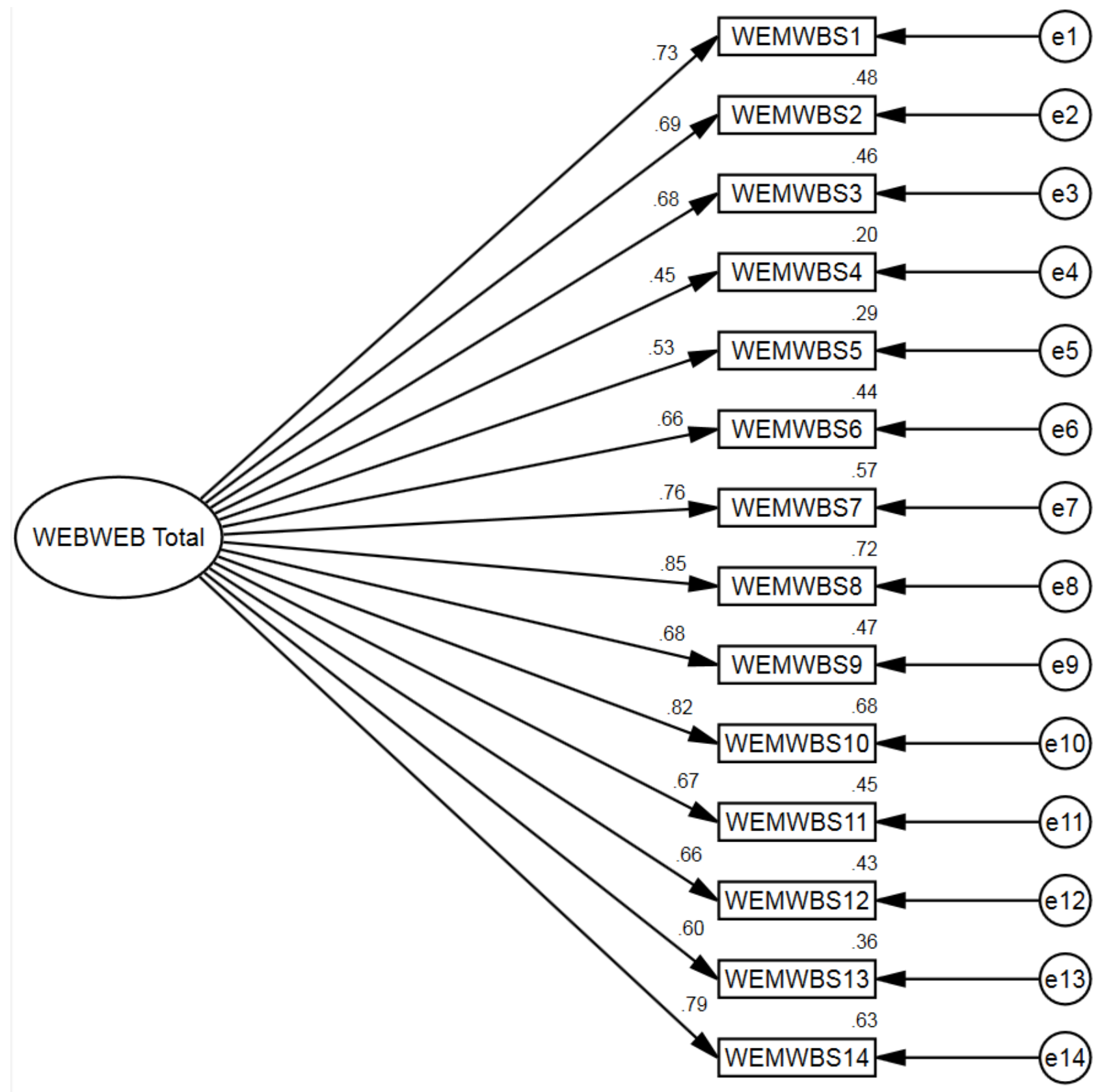

Figure 1. Standardized estimates for 14-Item WEMWBS. 


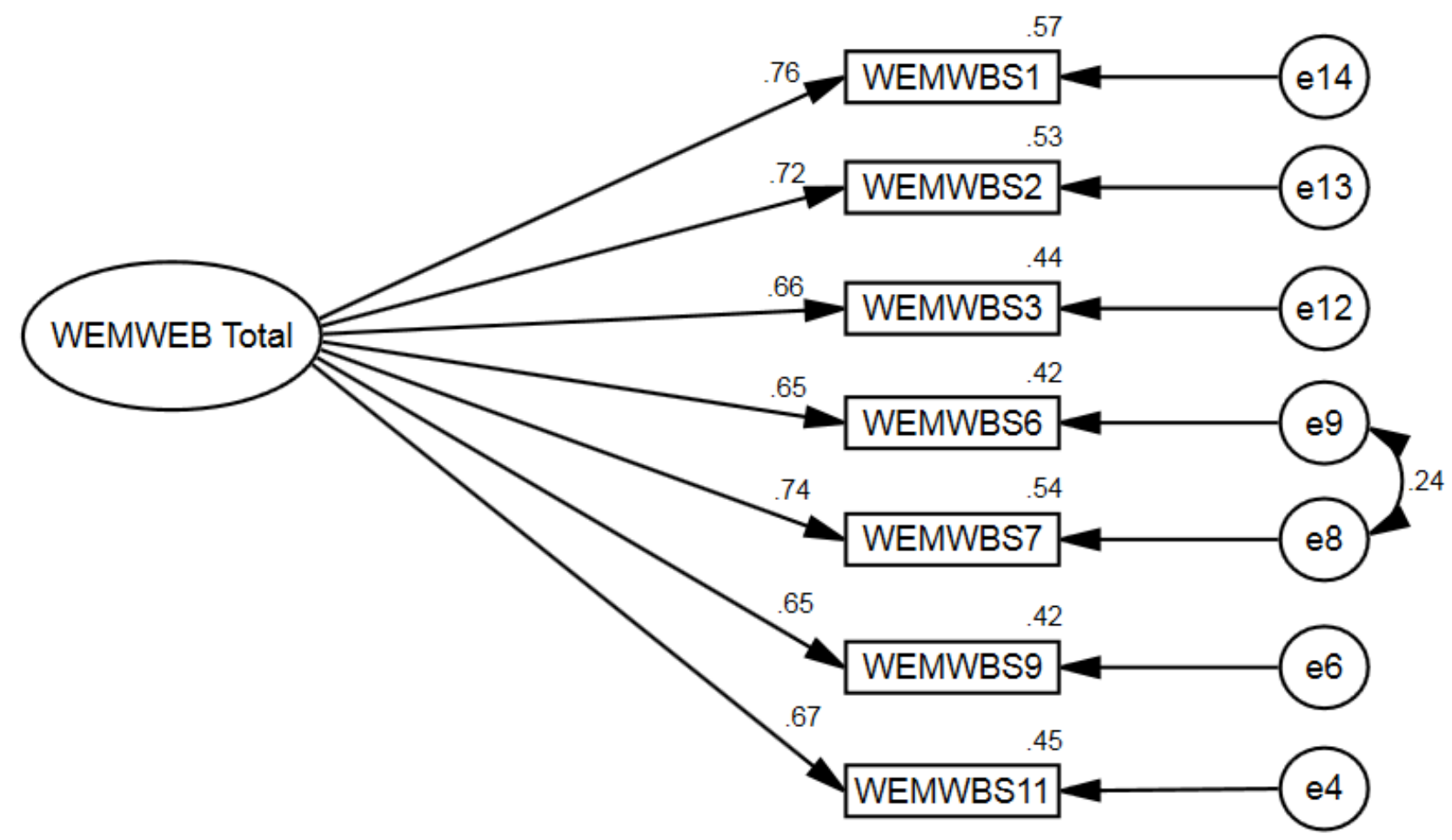

Figure 2. Standardized estimates for 7-Item SWEMWBS. 